\title{
Efficacy of Biportal Endoscopic Spine Surgery for Lumbar Spinal Stenosis
}

\author{
Dae-Jung Choi, DM, Ju-Eun Kim, MD* \\ Spine Center, Barun Hospital, Jinju, \\ *Department of Orthopedic Surgery, Andong Hospital, Andong, Korea
}

Background: Biportal endoscopic spine surgery (BESS) is a recent addition to minimally invasive spine surgery treatments. It boasts excellent magnification and fine discrimination of neural structures. Selective decompression with preservation of facet joints for structural stability is also feasible owing to access to the spinal canal and foramen deeper inside. This study has a purpose to investigate clinical benefits of BESS for spinal stenosis in comparison to the other common surgical treatments such as microscopic decompression-only (DO) and fusion and instrumentation (FI).

Methods: From December 2013 to March 2015, 30 cases of DO, 48 cases of Fl, and 66 consecutive cases of BESS for lumbar spinal stenosis (LSS) were enrolled to evaluate the relative clinical efficacy of BESS. Visual analog scale (VAS) for back pain and leg pain, postoperative hemoglobin, C-reactive protein (CRP) changes, transfusion, and postoperative complications were examined.

Results: All the patients were followed up until 6 months, and 98 patients (86.7\%) for 2 years. At the 6-month follow-up, VAS for back pain improved from 6.8 to $2.8,6.8$ to 3.2 , and 6.8 to $2.8(p=0.078)$ for BESS, DO, and FI, respectively; VAS for leg pain improved from 6.3 to $2.2,7.0$ to 2.5 , and 7.2 to $2.5(p=0.291)$, respectively. Two cases in the BESS group underwent additional foraminal decompression, but no fusion surgery was performed. Postoperative hemoglobin changes for BESS, DO, and FI were -2.5 , -2.4 , and $-1.3 \mathrm{~mL}$, respectively. The BESS group had no transfusion cases, whereas 10 cases (33.3\%) in D0 and 41 cases (85.4\%) in FI had transfusion $(p=0.000$ ). CRP changes for BESS, DO, and FI were $0.32,6.53$, and 6.00 , respectively, at day 2 postoperatively ( $p$ $=0.000$ ); the complication rate for each group was 8.6\% (two dural tears and one root injury), $6.7 \%$ (two dural tears), and 8.3\% (two dural tears and two wound infections), respectively.

Conclusions: BESS for LSS showed clinical results not inferior to those of the other open surgery methods in the short-term. Stable hemodynamic changes with no need for blood transfusion and minimal changes in CRP were thought to cause less injury to the back muscles with minimal bleeding. Foraminal stenosis decompression should be simultaneously conducted with central decompression to avoid an additional surgery.

Keywords: Lumbar vertebrae, Spinal stenosis, Minimally invasive surgical procedures, Endoscopy

There are several minimally invasive endoscopic spine procedures to treat lumbar spinal stenosis (LSS). ${ }^{1,2)}$ Due to technical barriers in handling the rigid endoscope and

Received August 10, 2018; Accepted October 21, 2018

Correspondence to: Dae-Jung Choi, DM

Spine Center, Barun Hospital, 7 Dongjin-ro, Jinju 52725, Korea

Tel: +82-55-790-3000, Fax: +82-55-790-3030

E-mail:djchoi9@hanmail.net limited availability of instruments, they have been only performed by experienced endoscopic surgeons. ${ }^{3)}$ In the meantime, open laminectomy alone or with lumbar spinal fusion has been the standard surgical method for degenerative spinal stenosis. However, it has been continuously associated with complications such as paraspinal muscle injury, excessive bleeding, and wound infection. ${ }^{4,5)} \mathrm{Re}-$ cently, favorable results of minimally invasive decompression surgery designed to overcome these issues have been reported in comparison to the open decompression or fu- 
sion surgery. ${ }^{6,7)}$ These reports can be considered as strong evidence of the superiority of the recent techniques for LSS by eliminating causative lesions and saving innocent structures for spinal stability. Biportal endoscopic spine surgery (BESS), a recent addition to minimally invasive spine surgery procedures, provides a wider range of view and allows for free handling of various instruments under a much clearer and magnified view. ${ }^{8,9)}$ The efficacy of BESS for LSS compared to other surgical treatments has not been reported because of it being relatively new and the short follow-up. In this study, we report the clinical results of BESS for LSS based on at least 2 years of follow-up.

\section{METHODS}

From December 2013 to March 2015, 84 cases of open decompression-only (DO) and fusion with instrumentation (FI) after decompression and 66 cases of the BESS were performed for lumbar spinal diseases. The inclusion criterion was LSS with or without grade I degenerative spondylolisthesis. We excluded LSS with potential instability, such as LSS with disc herniation, spondylolytic spondylolisthesis, and grade II or more degenerative spondylolisthesis. The surgical indication was the same for the three different methods regardless of severity and presence of combined foraminal stenosis. The patients with more than three-level LSS, however, were assigned to undergo DO rather than FI for preventing multilevel fusion and disability or BESS for shortening. DO or FI was performed from December 2013 to December 2014. BESS was performed from January 2015 to March 2015. Severity of spinal stenosis and presence of foraminal stenosis with or without spondylolisthesis did not affect the choice of surgical technique.

To evaluate patient satisfaction, the visual analog scale (VAS) for back pain and leg pain was used. BESS required the least amount of back muscle dissection so that the VAS score for back pain was evaluated in addition to VAS for leg pain in the assessment of successful decompression of causative lesions compressing the roots. To evaluate clinical efficacy, postoperative changes in hemoglobin and C-reactive protein (CRP) and cases of transfusion were compared among the three methods. These factors could serve as indirect indicators of the severity of back muscle injury. Postoperative complications such as dural tear, root injury, postoperative infection and epidural hematoma, were also investigated to overcome technical problems associated with the early learning period. Those factors could be comparable factors for technical risk or safety of performance. We conducted this study in com- pliance with the principles of the Declaration of Helsinki. The protocol of this study was reviewed and approved by the Institutional Review Board of Andong Hospital (IRB No. 2018-005). All patients were informed and allowed that their clinical information could be used only for medical study.

\section{Statistical Analysis}

Frequency data such as the sex ratio, transfusion, dural tear, root injury, postoperative wound infection, and epidural hematoma were analyzed using chi-square test or Fisher exact test. Continuous data such as differences in pre-, post- and following-up results in terms of VAS of back pain, VAS of leg pain, and CRP were analyzed using Wilcoxon signed-ranks test as nonparametric test because the sample size of DO $(n=30)$ and BESS $(n=35)$ were relatively small. Statistical difference in the average of continuous data among the three methods were evaluated using one-way analysis of variance test. The acceptance of the null hypothesis was dependent on the $p$-value greater than the significance level 0.05 . The statistical tool used was IBM SPSS ver. 21.0 (IBM Corp., Armonk, NY, USA).

\section{Surgical Techniques}

\section{Decompression-only}

Unilateral laminectomy bilateral decompression was performed under microscope. When approaching foraminal stenosis, contralateral decompression was performed using a curved foraminal Kerrison punch under a loupe because the deeper area of the contralateral foramen could be accessed easier just by bending of the surgeon's neck wearing a loupe and retracting back the muscle by a Taylor retractor rather than titling the operating table under a steep angle of the microscopic view. Bony structures of facets, by minimal dissection of back muscles, were preserved as much as possible for stability using partial laminectomy. Contralateral approach for sufficient decompression of the opposite side and contralateral foramen, however, needed to sacrifice the interspinous ligament and some part of the proximal and distal spinous process in the case of severe narrowing of the interspinous space due to the collapsed disc space. Flavectomy was performed to the attachment sites below the proximal lamina, lateral recess and distal part with distal laminectomy. A drain tube was inserted in every case before closing muscles and the skin.

\section{Fusion and instrumentation}

Decompression technique was the same as DO described above. After unilateral facetectomy, and the tip of the superior articular process was removed to expose the disc 
space. Disc space preparation was performed for a cage with laminectomized chip bone with or without bone substitutes. Instrumentation was proceeded using percutaneous screw fixation system under $\mathrm{C}$-arm. Irrigation was performed about every 20 minutes during the procedure; in particular, disc space irrigation before inserting bone graft and a cage was mandatory to prevent deep wound infection. Drain insertion and the rest of the procedure were the same as those of DO.

\section{Biportal endoscopic spine surgery}

The patient was placed in the prone position. Two standard entry points were made about $2 \mathrm{~cm}$ apart at the margin of the interlaminar space confirmed on the anteroposterior view. Subcutaneous fascia and muscles were split using a blunt dilator. Using a $0^{\circ}$ arthroscope, the targeted facet joint was identified with a freer elevator. Muscle detachment using a dilator in the interlaminar space before inserting of the arthroscope helped to secure sufficient visualization during the procedure. Fluent water flow over the inlets was checked to not increase the water pressure in the spinal canal and overcompress the dura which might increase the intracranial pressure. Natural pressure from a 3,000 $\mathrm{mL}$ saline bag at a level $50-80 \mathrm{~cm}$ higher than the level of the patient's back could be sustained. The pressure was about $25-30 \mathrm{mmHg}$ when using an infusion pump. After securing the water flow and catching the facet joint in front of the visual field, the rest of the procedure could proceed just as done in open surgery with the help of a high-speed burr, a Kerrison punch, and angled curettes.

To approach the contralateral side, sufficient working space for the arthroscope and certain working instruments was secured by partial resection of the base (10 o'clock at left side approach) and distal end (12 o'clock) of the spinous processes. During flavectomy of the contralateral side, the ligamentum flavum (LF) was detached or loosened from the margins of the medial and dorsal sides of the contralateral facet joint just as done on the ipsilateral side. Going forward, pushing an instrument under the tight LF could sever the dura or the nerve root; therefore, insertion of an instrument under the LF before securing the space under the LF was avoided. Considering dural central folding was hidden under epidural central fat tissue (Fig. 1), efforts were made to cross the midline between the LF and fat tissue rather than between the fat tissue and dura. Forceful detachment of LF at the midline from the dural central folding could make an incidental dural tear. Three of the four roots could be clearly observed in one segment during decompression. The exiting root of the ipsilateral side could not be easily observed. Muscle bleeding was controlled by a radiofrequency wand under low-voltage coagulation set. Intraspinal bleeding from the

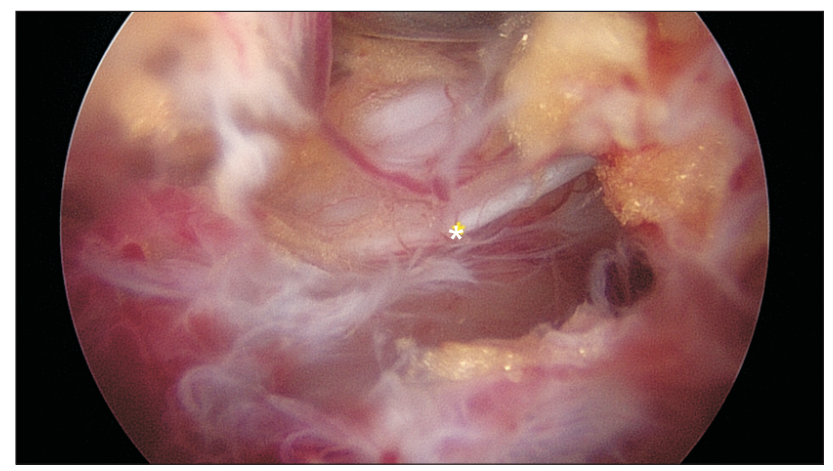

Fig. 1. Intraoperative arthroscopic view of the central folding (asterisk). Midline folding is hidden under the epidural fat and the ligamentum flavum. Care should be taken not to injure the structure when crossing the midline to decompress the contralateral side.

\section{Table 1. Demographics of the Study Groups}

\begin{tabular}{lcccc}
\multicolumn{1}{c}{ Variable } & BESS & DO & FI & p-value \\
\hline No. of cases & 35 & 30 & 48 & 0.279 \\
\hline Age (yr), mean \pm SD (range) & $65.4 \pm 11.8(36-85)$ & $65.2 \pm 12.0(33-84)$ & $65.0 \pm 8.8(48-81)$ & $19: 29$ \\
\hline Sex (male:female) & $14: 21$ & $17: 13$ & 39 & 0.118 \\
No. of levels & & & 9 & 0.290 \\
\hline 1 & 24 & 15 & 0 & 0 \\
\hline 2 & 11 & 9 & 3 & 0 \\
\hline
\end{tabular}

BESS: biportal endoscopic spine surgery, DO: decompression-only, Fl: fusion and instrumentation, SD: standard deviation. 
laminectomized bone edge was compressed by squashing a piece of bone wax on the bleeding sites. Bleeding from the epidural edge just after flavectomy from the small epidural vessels could be coagulated using a small sized (1.5$\mathrm{mm}$ ball tip) radiofrequency wand which is commonly used in wrist arthroscopy.

\section{RESULTS}

BESS was performed on 35 patients (male, 14; female, 21; age, $65.4 \pm 11.8$ years), DO on 30 patients (male, 17; female, 13; age, $65.2 \pm 12.0$ years), and FI on 48 patients (male, 19; female, 29; age, $65.0 \pm 8.8$ years) (Table 1). All the patients were followed up for 6 months for VAS for

Table 2. Comparison of Results among Groups

\begin{tabular}{|c|c|c|c|c|}
\hline Variable & BESS & DO & $\mathrm{FI}$ & $p$-value \\
\hline \multicolumn{5}{|l|}{ VAS } \\
\hline \multicolumn{5}{|l|}{ Back pain } \\
\hline Preoperative & $6.8 \pm 1.0$ & $6.8 \pm 1.2$ & $6.8 \pm 1.1$ & 0.988 \\
\hline PO 1 month & $3.0 \pm 0.8$ & $3.7 \pm 1.1$ & $4.0 \pm 0.9$ & 0.000 \\
\hline Follow-up 6 months & $2.8 \pm 1.0$ & $3.2 \pm 0.9$ & $2.8 \pm 0.8$ & 0.078 \\
\hline \multicolumn{5}{|l|}{ Leg (calf) pain } \\
\hline Preoperative & $6.3 \pm 1.1$ & $7.0 \pm 1.1$ & $7.2 \pm 0.9$ & 0.001 \\
\hline PO 1 month & $2.4 \pm 0.8$ & $2.9 \pm 0.9$ & $3.0 \pm 0.9$ & 0.012 \\
\hline Follow-up 6 months & $2.2 \pm 0.8$ & $2.5 \pm 0.7$ & $2.5 \pm 0.8$ & 0.291 \\
\hline \multicolumn{5}{|l|}{ Hemoglobin change (MCV, mL) } \\
\hline Preoperative & $13.5 \pm 1.2$ & $13.8 \pm 1.8$ & $13.3 \pm 1.1$ & 0.331 \\
\hline Difference at $\mathrm{PO} 2$ days & $-2.5 \pm 0.7$ & $-2.4 \pm 2.0$ & $-1.3 \pm 1.1$ & 0.000 \\
\hline Difference at P0 6-7 days & $-1.5 \pm 0.7$ & $-2.0 \pm 1.3$ & $-1.9 \pm 1.3$ & 0.259 \\
\hline No. of patients with transfusion & 0 & $10(33.3)$ & $41(85.4)$ & 0.000 \\
\hline Transfusion volume within the relevant patients $(\mathrm{mL})$ & 0 & $568.0 \pm 265.2$ & $581.5 \pm 207.9$ & 0.000 \\
\hline \multicolumn{5}{|l|}{ C-reactive protein change } \\
\hline Preoperative & $0.19 \pm 0.53$ & $0.26 \pm 0.75$ & $0.28 \pm 0.98$ & 0.727 \\
\hline Difference at $\mathrm{PO} 2$ days & $0.32 \pm 0.79$ & $6.53 \pm 5.45$ & $6.00 \pm 4.60$ & 0.000 \\
\hline Difference at PO 1 week & $0.17 \pm 0.70$ & $1.68 \pm 1.92$ & $2.02 \pm 1.54$ & 0.000 \\
\hline Difference at PO 2 weeks & $0.41 \pm 2.40$ & $0.82 \pm 1.53$ & $0.63 \pm 1.27$ & 0.680 \\
\hline Total case of complications & $3(8.6)$ & $2(6.7)$ & $4(8.3)$ & - \\
\hline Dural tear & 2 & 2 & 2 & \\
\hline Root injury & 1 & 0 & 0 & \\
\hline Wound infection & 0 & 0 & 2 & \\
\hline Superficial & 0 & 0 & 1 & \\
\hline Deep & 0 & 0 & 1 & \\
\hline Hematoma & 0 & 0 & 0 & \\
\hline
\end{tabular}

Values are presented as mean \pm standard deviation or number (\%).

BESS: biportal endoscopic spine surgery, DO: decompression-only, Fl: fusion and instrumentation, VAS: visual analog scale, MCV: mean corpuscular volume, PO: postoperative. 
back pain and leg pain. Ninety-eight patients (86.7\%) were followed up for at least 2 years; i.e., 33 patients (94.3\%), $24.5 \pm 3.7$ months after BESS; 25 patients (83.3\%), $30.5 \pm$ 2.4 months after DO; and 40 patients (83.3\%), $34.9 \pm 3.6$ months after FI.

At the preoperative assessment and 1-month and 6-month follow-up, VAS for back pain improved from 6.8 \pm 1.0 via $3.0 \pm 0.8$ to $2.8 \pm 1.0$ with BESS, $6.8 \pm 1.2$ via 3.7 \pm 1.1 to $3.2 \pm 0.9$ with $\mathrm{DO}$, and $6.8 \pm 1.1$ via $4.0 \pm 0.9$ to $2.8 \pm 0.8$ with FI (1-month follow-up, $p=0.000$; 6-months follow-up, $p=0.078)$. VAS for leg pain improved from 6.3 \pm 1.1 via $2.4 \pm 0.8$ to $2.2 \pm 0.8,7.0 \pm 1.1$ via $2.9 \pm 0.9$ to 2.5 \pm 0.7 , and $7.2 \pm 0.9$ via $3.0 \pm 0.9$ to $2.5 \pm 0.8(p=0.012, p=$ 0.291 ) with BESS, DO, and FI, respectively.

Postoperative hemoglobin changes were $-2.5 \pm 0.7$, $-2.4 \pm 2.0$, and $-1.3 \pm 1.1 \mathrm{~mL}$ after BESS, DO, and FI, respectively. Patients with BESS had no transfusion cases but transfusion was needed in 10 cases (33.3\%) after DO and $41(85.4 \%)$ cases after FI $(p=0.000)$. CRP changes were $0.32 \pm 0.79,6.53 \pm 5.45$, and $6.00 \pm 4.60$ on postoperative day 2 after BESS, DO, and FI, respectively $(p=0.000)$. The complication rate was $8.6 \%$ after BESS (two cases of dural tear and one case of root injury), 6.7\% after DO (two cases of dural tear), and $8.3 \%$ after FI (two cases of dural tear and two cases of wound infection) (Table 2). During the 2-year follow-up, two cases in patients with BESS needed additional foraminal decompression at the previously central decompressed levels, which was performed using BESS via the extraforaminal approach (Fig. 2).

\section{DISCUSSION}

For decades, open decompression has been widely thought to be the gold standard treatment for LSS. But extensive soft tissue dissection can cause paravertebral muscle atrophy and might result in heaviness and pain in the back for a long time. ${ }^{4)}$ Resection of interspinous ligaments for better surgical vision also may cause instability and it needs instrumentation. ${ }^{10,11)}$ In recent trials, less invasive approaches to reduce negative hazards and permit accessibility for bilateral decompression have been introduced; e.g., microendoscopic surgery through a tubular retractor through paravertebral or midline approach and modified spinous process osteotomy. ${ }^{12-14)}$ All surgical treatments for LSS should focus on "reducing sacrifice of the negative components" including skin, paravertebral muscles, and interspinous ligaments simultaneously attempting to surgically remove the causative structures of hypertrophied LF and outgrowing spur of superior articular processes. From these points of view, endoscopic trials for LSS have been introduced in recent years. Although it has outstanding merits including minimal blood loss, short operating time, minor complications, and early discharge, ${ }^{1)}$ technically it is not easy to perform and covers limited indications. Hence, conventional endoscopic spine surgery through one portal was recommended only for experienced endoscopic surgeons according to the International Society for Minimal Intervention in Spinal Surgery guidelines (http://www. ismiss.com). ${ }^{3)}$

BESS uses two portals instead of one. One of them is for an arthroscope for viewing and the other for surgi$\mathrm{cal}$ instruments. This allows for a wider range of view with free handling of basic instruments in open spine surgery, which can make it possible to decompress a stenotic lesion widely and safely with preservation of the paravertebral muscles and interspinous ligaments for the posterior
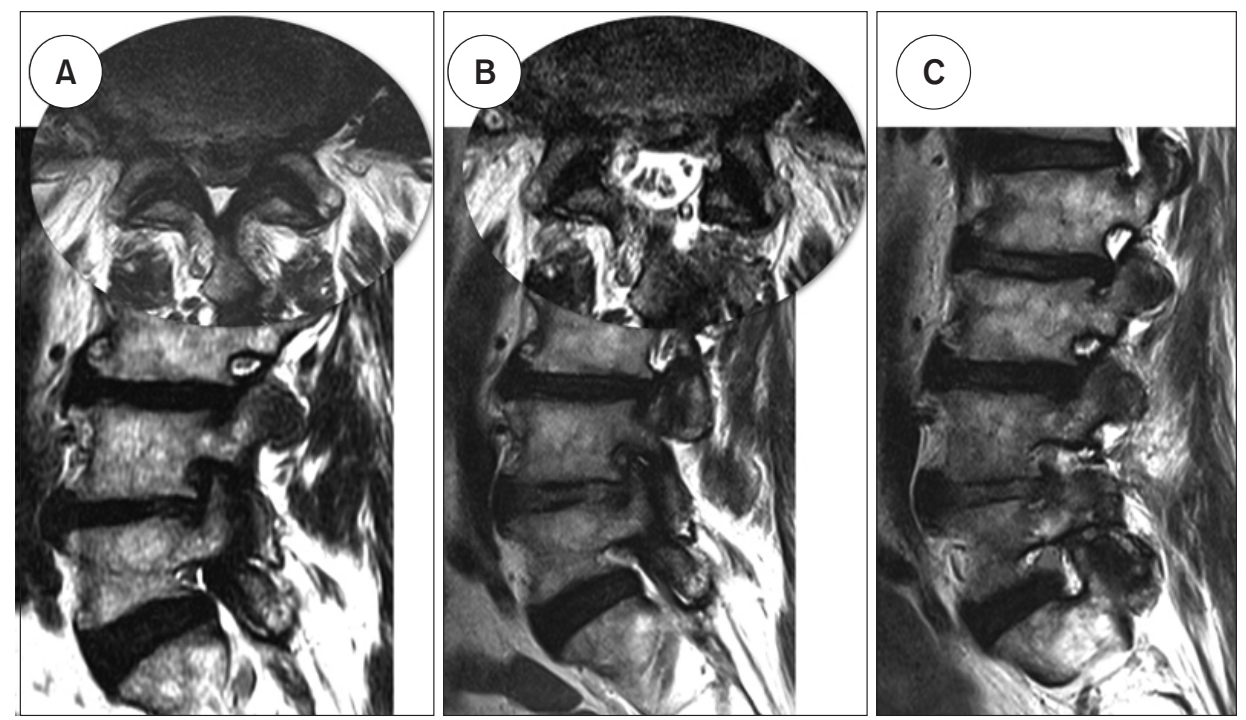

Fig. 2. Additional foraminal decompression for remnant foraminal stenosis. (A) The preoperative magnetic resonance imaging scan of a 74-year-old male patient showed central stenosis at the L4-5 combined with foraminal stenosis on the right side. (B) Central decompression using biportal endoscopic spine surgery (BESS) was successfully performed on remnant foraminal stenosis. (C) Additional foraminal decompression using extraforaminal approach with BESS was performed at the 7-month follow-up. 
integrity of the spinal column. The results of this study showed that patients who underwent BESS had lower back heaviness and back pain especially in the early healing period 1 month after the surgery. It might be because of preservation of the paravertebral muscles, which was quite different from the DO or FI procedures. BESS also showed comparable results to those of the other procedures in terms of leg and back pain improvement, which means that it could be a possible alternative technique for sufficient decompression of the dura and roots in LSS.

CRP is an acute inflammatory protein that increases at sites of infection or inflammation. There is now growing evidence that CRP plays important roles in inflammatory processes. ${ }^{15,16)}$ On postoperative one or two, the CRP level was significantly lower in the patients with BESS, which was normalized within 7 days. This means that tissue destruction was much less in the BESS procedure and possible inflammatory debris could be irrigated better during the procedure compared with the other procedures. CRP changes after all three procedures should not be considered as the occurrence of infection in the early postoperative period. However, early normalization in the BESS patients strongly supports that intravenous antibiotics could be necessary only within 1 or 2 days for prophylaxis because the endoscopic surgery involves lots of saline irrigation and small skin incisions for inlets. Postoperative hemoglobin level was two points lower than the preoperative level because of hydration by saline during surgery. Conversely, the FI procedure caused a less decrease possibly because of the higher frequency of transfusion. In the BESS patients, there was no transfusion required compared to $33 \%$ and $85 \%$ for DO and FI, respectively. Decompression for symptomatic relief in the patients with LSS without transfusion was supposed to be recommendable during BESS in the presence of multi-level stenosis or senile patients with hemodynamic instability.

The neurological complication rate for BESS was $8.6 \%$ with two cases of dura tear and one case of contralateral root injury associated with technical problems. It was related to the learning curve period and the preventive strategies in the procedure were described in the surgical technique. ${ }^{17,18)}$ To reduce the occurrence of this technical issue, the most important factor is to keep the surgical field clear by blocking epidural bleeding. In the surgical technique described above, fluent water flow and bleeding control from edged bone or epidural small vessels were ensured before proceeding with flavectomy or laminectomy especially on the contralateral side. If the bleeding cannot be controlled with every effort, lowering the diastolic blood pressure to around $100 \mathrm{mmHg}$ can be helpful in some cases. However, increasing the saline pressure by raising the height of the saline bag or squeezing the bag is not recommended. This is because if there is no good outflow of saline, the surgical field becomes turbid with small bleeds even with a high input pressure of saline.

During the learning curve, there were similar disturbances such as prolonged operating time for one level decompression due to the turbid surgical field by small bleeds and frequent dura tears due to insufficient information on the endoscopic view and surgical anatomy. There was also incomplete decompression especially in the patients with central stenosis and foraminal stenosis due to unskilled decompression. ${ }^{7)}$ Radicular pain from foraminal stenosis could be easily masked by claudication with shorter distance walking. However, when the patients tried to walk longer distances after central decompression, the fatigue and buttock pain because of foraminal stenosis were attenuated and became intolerable.

This study has some limitations. First, there were too few cases for each procedure to evaluate other technical complications. Second, because of the technical issues with BESS performed in the early learning period, there were higher neurological complication rates, and some patients were dissatisfied with decompression. Third, fusions involving more than three levels were not performed to avoid the risk of early revision surgery due to nonunion, wound infection, longer rehabilitation, and screw loosening in senile patients. These patients were intentionally assigned to the DO procedure. Therefore, the clinical scores of DO patients were not supposed to be lower than those of BESS patients with shorter level decompression. The differences in the number of level of surgery among procedures might also have biased the clinical results. Therefore, prospective, head-to-head studies with matched levels and mid- and long-term follow-ups are needed to clarify the efficacy of BESS.

BESS as an alternative treatment for LSS showed clinical efficacy comparable to that of the other open surgeries in the short-term follow-up. Stable hemodynamic results without transfusion that led to less amount of bleeding because of minimal back muscle dissection through two inlets could be another merit of this technique. The presence of combined foraminal stenosis should be carefully evaluated before surgery, and foraminal decompression should be included during surgical planning to avoid additional surgery in the future.

\section{CONFLICT OF INTEREST}

No potential conflict of interest relevant to this article was 
reported.

\section{REFERENCES}

1. Polikandriotis JA, Hudak EM, Perry MW. Minimally invasive surgery through endoscopic laminotomy and foraminotomy for the treatment of lumbar spinal stenosis. J Orthop. 2013;10(1):13-6.

2. Wong AP, Smith ZA, Lall RR, Bresnahan LE, Fessler RG. The microendoscopic decompression of lumbar stenosis: a review of the current literature and clinical results. Minim Invasive Surg. 2012;2012:325095.

3. Birkenmaier C, Komp M, Leu HF, Wegener B, Ruetten S. The current state of endoscopic disc surgery: review of controlled studies comparing full-endoscopic procedures for disc herniations to standard procedures. Pain Physician. 2013;16(4):335-44.

4. Hu ZJ, Fang XQ, Zhou ZJ, Wang JY, Zhao FD, Fan SW. Effect and possible mechanism of muscle-splitting approach on multifidus muscle injury and atrophy after posterior lumbar spine surgery. J Bone Joint Surg Am. 2013;95(24):e192(1-9).

5. Huang YH, Ou CY. Significant blood loss in lumbar fusion surgery for degenerative spine. World Neurosurg. 2015;84(3):780-5.

6. Choi DJ, Jung JT, Lee SJ, Kim YS, Jang HJ, Yoo B. Biportal endoscopic spinal surgery for recurrent lumbar disc herniations. Clin Orthop Surg. 2016;8(3):325-9.

7. Choi DJ, Choi CM, Jung JT, Lee SJ, Kim YS. Learning curve associated with complications in biportal endoscopic spinal surgery: challenges and strategies. Asian Spine J. 2016;10(4):624-9.

8. Scholler K, Alimi M, Cong GT, Christos P, Hartl R. Lumbar spinal stenosis associated with degenerative lumbar spondylolisthesis: a systematic review and meta-analysis of secondary fusion rates following open vs minimally invasive decompression. Neurosurgery. 2017;80(3):355-67.

9. Forsth P, Olafsson G, Carlsson T, et al. A randomized, con- trolled trial of fusion surgery for lumbar spinal stenosis. N Engl J Med. 2016;374(15):1413-23.

10. Tai CL, Hsieh PH, Chen WP, Chen LH, Chen WJ, Lai PL. Biomechanical comparison of lumbar spine instability between laminectomy and bilateral laminotomy for spinal stenosis syndrome: an experimental study in porcine model. BMC Musculoskelet Disord. 2008;9:84.

11. Hartmann F, Janssen C, Bohm S, Hely H, Rommens PM, Gercek E. Biomechanical effect of graded minimal-invasive decompression procedures on lumbar spinal stability. Arch Orthop Trauma Surg. 2012;132(9):1233-9.

12. Pao JL, Chen WC, Chen PQ. Clinical outcomes of microendoscopic decompressive laminotomy for degenerative lumbar spinal stenosis. Eur Spine J. 2009;18(5):672-8.

13. Takaso $M$, Nakazawa $T$, Imura $T$, et al. Less invasive and less technically demanding decompressive procedure for lumbar spinal stenosis: appropriate for general orthopaedic surgeons? Int Orthop. 2011;35(1):67-73.

14. Mikami Y, Nagae M, Ikeda T, Tonomura H, Fujiwara H, Kubo T. Tubular surgery with the assistance of endoscopic surgery via midline approach for lumbar spinal canal stenosis: a technical note. Eur Spine J. 2013;22(9):2105-12.

15. Gewurz H, Mold C, Siegel J, Fiedel B. C-reactive protein and the acute phase response. Adv Intern Med. 1982;27:345-72.

16. Sproston NR, Ashworth JJ. Role of C-reactive protein at sites of inflammation and infection. Front Immunol. 2018;9:754.

17. Turner JA, Ersek M, Herron L, Deyo R. Surgery for lumbar spinal stenosis: attempted meta-analysis of the literature. Spine (Phila Pa 1976). 1992;17(1):1-8.

18. Nowitzke AM. Assessment of the learning curve for lumbar microendoscopic discectomy. Neurosurgery. 2005;56(4):755-62. 